\title{
REFLECTION AND TRANSMISSION OF ELECTRIC WAVES BY RESONATOR GRATINGS.
}

\author{
Remarks on a Paper Under This Title, by F. C. Blake \\ and C. R. Fountarn.
}

By Clemens Schaefer.

I $\mathrm{N}$ a research, ${ }^{1}$ which has just been published, Messrs. Blake and 1 Fountain have investigated the reflection and transmission of electric waves by systems of resonators.

The method which they use is similar to that which Garbasso, ${ }^{2}$ Aschkinass and $\mathrm{I}^{3}$ have employed; we will speak later of the changes they have introduced. Their results confirm in part those previously obtained by the above-mentioned authors. Along with other work, $\mathrm{I}^{4}$ published some of their results in an article under the title, "Concerning the Selective Properties of Resonator Gratings," in the Annalen der Physik, in January, I905. This article has apparently not come to the notice of Messrs. Blake and Fountain. At least I can find no evidence that it has, although in the article by Pätzold, cited by them in their appendix, my work is frequently mentioned.

This is my reason for giving the work of Messrs. Blake and Fountain somewhat closer consideration, and especially since their method, in those points where it differs from that of Aschkinass and myself, is by no means an improvement, but to my mind at least is even poorer.

As Garbasso first showed, the resonator gratings here used possess selective properties for electric waves ; i. e., they reflect to a considerable extent only those waves whose period of vibration

${ }^{1}$ F. C. Blake and C. R. Fountain, Phys. Rev., XXIII., p. 257, 1906.

${ }^{2}$ A. Garbasso, Atti Acc. di Torino, XXVIII., pp. 470 and 816,1893 . Journal de Physique, XXII., p. 259, I893.

${ }^{3}$ E. Aschkinass and C. Schaefer, Ann. d. Phys., V., p. 489, I 90 I.

${ }^{4}$ C. Schaefer, Ann. d. Phys., XVI., p. I06, 1905. 
does not differ greatly from their own. Two methods present themselves for the study of the relation between reflection, with its corresponding transmission, and the wave-length:

I. We may use a single resonator grating, which remains unchanged during the experiment. In this case we must use a variable exciter and receiver to give us the necessary wave-lengths within the limits over which we wish to study the extent of reflections or transmission. This method is exactly that which is used in the corresponding measurements in optics. This method seems to me for certain reasons to have the advantage over the one to follow.

II. We work with a single wave-length, $i$. e., with an unchanging exciter and receiver, varying instead the resonator gratings, i. e., the length of the resonators. With this method, too, which in a certain sense is only a reversal of the previous one, we may obtain unimpeachable results. By this method, for example, the investigations of Garbasso, and

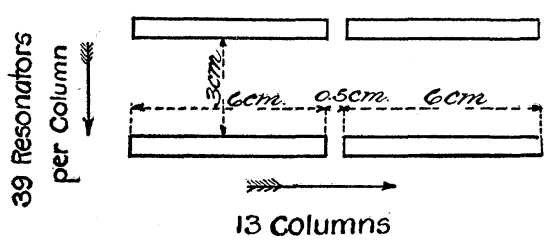

Fig. 1. Aschkinass-Schaefer were carried out. Messrs. Blake and Fountain have also used this second method, but in a way that is not entirely free from objection.

I will now try to make this clear. The characteristic point in the method used is the variation of the resonator grating. Let us see how this was done in this investigation. According to the table, page 265 , the authors start out, for example, in their curves $T$ and $R$ of Fig. 8, with a grating, the structure of which can be seen from the accompanying figure.

This grating they change by the gradual decrease of the resonators, all other dimensions of the apparatus remaining unaltered.

There are objections to this method, although at first sight it is apparently correct. In order to make this clear, consider the following: The power of a resonator grating to reflect, and consequently too, to transmit, depends not only on the length of the resonators but also on their number and their arrangement. So gratings made of resonators of different lengths are in general not at all comparable. That is, the question which rises in using the 
second method is this: How must resonator gratings, consisting of resonators of different lengths, be arranged, in order to be comparable with another? The answer to this question is easy, if we for a moment consider the relations holding when we employ the first method, which, as already stated, is entirely analogous to the method used in the corresponding optical measurements. Here the grating stays unchanged during the experiment, while the wave-length is varied. If we pass from a wave-length $\lambda_{1}$ to a second $\lambda_{2}$, the corresponding linear dimensions of the grating have changed in the proportion of $\lambda_{2} / \lambda_{1}$, using as a unit the new wavelength $\lambda_{2}$. And here we see the answer to our question: In using the second method it is not sufficient to change the length of the resonators alone, but all corresponding dimensions of the grating must be changed in the same ratio. Only thus can we obtain comparable relations, as in the case of those optical experiments where we meet selective reflections, and in this way we get exactly these relatively simple relations. The error in the method of Blake and Fountain is that they did not notice this fact, although Aschkinass and I laid especial emphasis upon it.

This explanation makes it at once clear how much simpler the first method is, because this particular difficulty does not enter into it at all. Hence the curves of Messrs. Blake and Fountain are quite complicated, and cannot well be compared with those of the previous investigators, and it is not possible to apply the ordinary dispersion formulæ to them directly as would be the case with the curves of Aschkinass and myself. In short, Blake and Fountain obtained, on account of the construction of their gratings, complicated relations instead of relatively simple ones.

I have mentioned already that many of the results obtained by Messrs. Blake and Fountain are only substantiations of the work of previous authors. I will now make this clear in a special case. Blake and Fountain discuss this problem: How does the length of the resonators corresponding to maximal resonance change, when the elements of the grating are brought closer together? They find, that a diminution of the distance between the resonators in a direction at right angles to the electric force requires a greater resonator length to produce maximal resonance. According to their results, 
within the limits investigated, a diminution of the distance in a direction parallel to the vector of electric force makes no difference at all. From the standpoint of the first method, this means that in the first case the point of maximal resonance is changed so that it occurs at a smaller wave-length, while in the second case it is unaffected.

I discussed this same question in the article previously mentioned, and studied the phenomena by the first method, which seems certainly better on account of its simplicity. The results I obtained are these: (I) A diminution of the distance in a direction at right angles to the electric vector changes the maximal resonance to a point of smaller wave-length. This is the same result as obtained by Blake and Fountain. (2) A decrease of the distance in a direction parallel to the electric force changes the maximal resonance to a point of greater wave-length. This result is exactly opposite to that of Blake and Fountain; however this contradiction is easily explained, since I varied the distances within wider limits than they. Further it must not be forgotten that, strictly speaking, the curves of Blake and Fountain are not at all comparable with mine. The fact that they did not find the change in the point of maximal resonance in the second case, is of itself a proof of the unsuitability of their method. Moreover, according to the explanation which Blake and Fountain put forward for the change in the first case, one would also expect a change in the second. Consider this explanation for a moment. They say, page 268 : " that the resonance length should decrease with increasing distance between the strips was to be expected; for since at any instant the currents in the resonators are all in the same direction, the effect of induction is reduced by decreasing the distance between them, hence in order to respond to a given wave the capacity of the resonators must be increased, that is, they must be lengthened."

I cannot understand this explanation because of its inexactness o statement. What is meant by "the effect of induction"? The only factor concerning the induction, which can be changed through a change in the distance between the resonators, is the coefficient of mutual induction. As I previously showed, ${ }^{1}$ however, this change 
would, in the first case, have an effect exactly opposite to the observed; while in the second case, on the other hand, the theoretical changes would correspond with those observed by myself. These points make it clear that the explanation in the first case, by means of the coefficient of mutual induction, is not complete. We must rather take into account that a change in the distance between the resonators also changes in general their capacity. The change in capacity, however, causes in both cases a change in the point of maximum resonance corresponding to the experimental results. That is, the explanation of Messrs. Blake and Fountain is certainly, if not absolutely wrong, at least incomplete.

I pass now to the consideration of a phenomenon which Messrs. Blake and Fountain discovered, and to which they gave the name of "Extra transmission." They met this phenomenon in the case of resonator gratings glued to glass plates. Essentially it is this, that such a resonator grating glued to glass, under certain conditions, transmits more, and consequently reflects less, than the glass plate alone. The authors explain this remarkable phenomenon by saying that the glass plate and resonators may be a medium with a variable index of refraction, and that, according to the optical dispersion formulæ, under certain conditions the index of refraction may become smaller than that of the glass itself. Although, as I have already shown, the optical dispersion formulæ are not here applicable entirely without restriction, still there is no objection to be raised to this explanation, provided the phenomenon in question is a fact. I must say, however, that the description of Blake's and Fountain's experiments has not convinced me of the reality of the phenomenon. My reasons are: first, that none of the earlier experimenters found this phenomenon, although, according to Blake and Fountain, it is quite marked. In particular, Aschkinass and I did not find this to happen. Hence in this latter investigation we must assume quite large errors of observation. Now, such lárge errors are surely here out of the question, as is easily seen from the excellent correspondence of our measurements of the dielectric constant with those of the other investigators. Moreover, I have repeated the measurements of Blake and Fountain in question, reproducing their arrangement of apparatus. The only change I made was to 
restrict the wave-flow by making it pass through two diaphragms, with openings of $24 \times 32 \mathrm{~cm}$. The resonator grating used was of the same size. It only remains to be said that the area of the resonator grating was smaller than the mouth of the concave mirror used. With this apparatus I was likewise unable to find any "extra transmission." More than that, the grating always transmitted less energy than the glass plate alone. If the diaphragms were removed, the transmittivity of the resonator grating increased; if the rays were made divergent, by moving the exciter a bit from the focus of the concave mirror, the transmittivity increased still more. Now this result is an approximation of the phenomenon of "extra transmission" ; but I did not succeed in verifying the observations of Blake and Fountain.

The question is, must we accept the explanation of Blake and Fountain, or can we give another. To answer this, it must be kept in mind, that these authors did not use diaphragms to restrict the wave flow; moreover the area of their resonator grating was larger than that of their parabolic mirror. Now, if we assume that the original electric waves were not exactly parallel, but slightly divergent, it would actually be possible to observe a phenomenon like " extratransmission." For the individual resonators of the grating would be set in vibration by the electric wave striking them, and thus would become themselves centers of spherical waves going out in all directions. Now, since in the experiments of Blake and Fountain the grating had a larger area than the concave mirror, in the focus of which the receiver is placed, "side waves" from the resonators on the border of the grating could reach the receiver when the grating was interposed, while the interposition of the glass plate alone let only direct waves reach the receiver. Under certain conditions these. "side waves" may become so marked, that putting the grating in the path of the wave would actually give an increase of the amount of energy reaching the receiver. As a matter of fact, M. Paetzold observed this very thing in the case of divergent radiation without interposition of diaphragms with gratings, whose area was larger than the mouth of the mirrors he used. This is the explanation of the results of my repetition of the experiments of Blake and Fountain. As soon as the diaphragms are taken away, 
we find an increase of transmittivity, which is, and ought to be, under proper experimental conditions, constant. The increase above the normal is still greater if we intentionally make the paths of the radiation divergent.

I do not yet say, that the "extra transmission" of Blake and Fountain is likewise due to poor experimental arrangements. But so long as the above objections to the experimental arrangement of the authors are not refuted, there is no reason for the assumption of a nere phenomenon.

Breslau, Physical Laboratory of the University, January, I907. 